\title{
Lateral nano-Newton force-sensing piezoresistive cantilever for microparticle handling
}

\author{
T Chu Duc, J F Creemer and P M Sarro
}

Delft University of Technology, DIMES, Electronic Components, Technology and Materials Laboratory, Delft PO Box 5053, 2600 GB Delft, The Netherlands

E-mail: t.chuduc@dimes.tudelft.nl

Received 29 November 2005, in final form 30 November 2005

Published 9 May 2006

Online at stacks.iop.org/JMM/16/S102

\begin{abstract}
A lateral force-sensing piezoresistive cantilever is presented that can be used to evaluate the impact force between micro-handling tools and microparticles in the nano-Newton range. The $500 \mathrm{~nm}$ thick piezoresistive sensors are made from epitaxial silicon on single crystal silicon. The cantilevers are fabricated using bulk micromachining and are $300 \mu \mathrm{m}$ long, $10 \mu \mathrm{m}$ high and $14 \mu \mathrm{m}$ wide. The applied force on this sensor is parallel to the wafer surface. This structure can eliminate the effect of the vertical force, increasing the sensitivity and accuracy of the system. The force resolution of the implemented sensor is $98 \mathrm{~V} \mathrm{~N}^{-1}$ with a minimum detectable force of $5 \mathrm{nN}$.
\end{abstract}

(Some figures in this article are in colour only in the electronic version)

\section{Introduction}

The development of nano-Newton force sensors for manipulating microparticles or living cells has become a great technological challenge for advanced microassembly and future living cell surgery. A lateral force-sensing cantilever can be used for force feedback on micro-probing, manipulation, positioning, cell separation as well as for minimally invasive surgery and micro-assembly.

The bending of the cantilever is related to the applied force. By monitoring the displacement the amplitude of the applied force can be detected. Several sensing methods can be used to detect forces: piezoresistive, capacitive and optical/laser detection. The optical method, mostly used in atomic force microscopy (AFM), is based on focusing a laser spot on the cantilever and then monitoring the motion of the reflected light using a photodiode. This method is very powerful in measuring small displacements, but it requires a highly accurate alignment of the optical system. The tip of the cantilever should be larger than the laser spot size. The method therefore becomes difficult when the size of the cantilever is decreased [1].

The capacitive method is based on the capacitance change when the structure is deformed and is the most widely used method in microaccelerometers and sensors for harsh environments. The capacitive structures, however, need to be completely isolated between two electrodes; so normally silicon-on-insulator (SOI) wafers are employed. Instead of SOI, trench isolation can be used [2]. However, it is difficult to control the etching area to obtain a completely isolated structure. Moreover, the capacitive method requires complicated electronic circuits.

Piezoresistive transducers translate a force into a change in the value of a resistor. They are widely employed as sensing elements in pressure sensors, accelerometers, and cantilevers used as nano mechanical sensors [3], binding force sensors [4] and bio-chemical mass sensors [5]. Recently, developments in the fabrication process have resulted into submicron piezoresistive cantilevers with $\mathrm{pN}$ [6] and even fN [7] resolution. However, most of these force sensors are limited in their application because they employ vertical structures [3, 4, 6, 8]. Vertical structures can only detect the forces perpendicular to the wafer surface.

Chui et al [9] presented an independent detection of vertical and lateral forces sensor. But they used oblique implantation, a rather special technique, in order to produce resistors on the vertical sidewalls of the cantilever. 

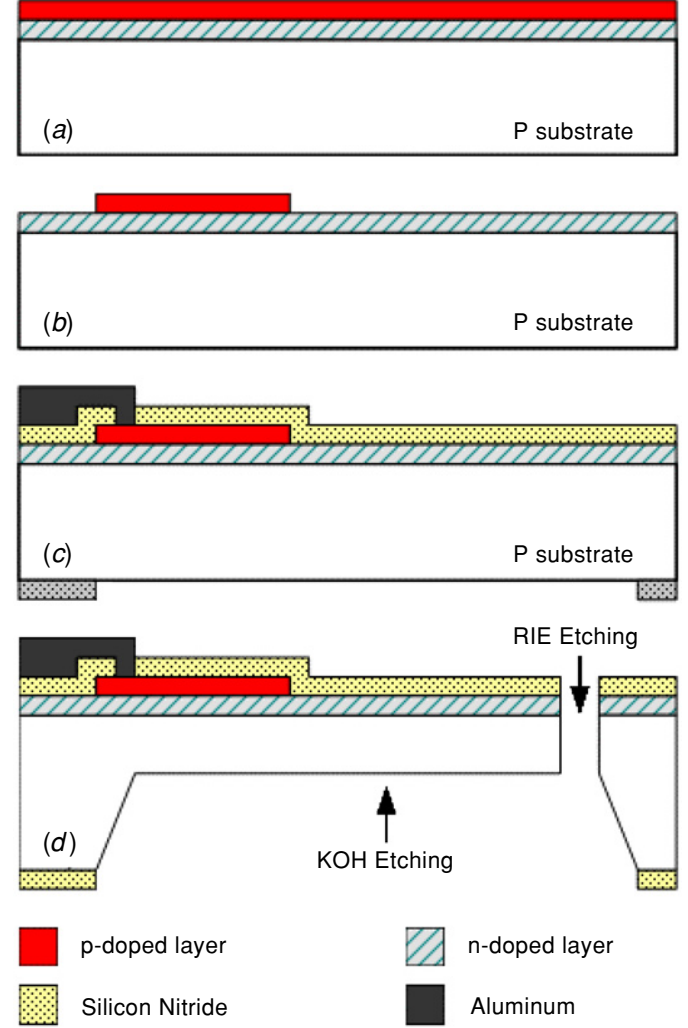

Figure 1. Schematic view of the fabrication process: $(a)$ epitaxial growth; $(b)$ piezoresistors definition; $(c)$ insulation and metallization; $(d)$ cantilever release.

This paper presents a novel lateral force-sensing piezoresistive cantilever providing nano-Newton sensitivity and the possibility to be combined with handling tools. By using two sensing resistors located on both sides of the cantilever and the Wheatstone bridge configuration, the piezoresistive cantilever is capable of detecting the lateral bending caused by the applied forces parallel to the wafer surface. The cantilever is made on regular silicon wafers and the resistors are made by employing epitaxial growth. Moreover, the fabrication method is compatible with CMOS processes.

\section{Fabrication}

The lateral force sensor consists of a p-type silicon cantilever on which p-type silicon piezoresistors are integrated. The cantilevers are 300 to $500 \mu \mathrm{m}$ long, 10 to $18 \mu \mathrm{m}$ wide, and 10 to $20 \mu \mathrm{m}$ thick and they contain 1 to $2 \mu \mathrm{m}$ wide and 47 to $73 \mu \mathrm{m}$ long built-in piezoresistors.

The fabrication process is based on the DIMES-03 bipolar process [10] and it is schematically depicted in figure 1 . The starting material is a p-type $\left(\begin{array}{lll}1 & 0 & 0\end{array}\right)$ silicon wafer with a $1 \mu \mathrm{m}$ thick n-type epitaxial layer, with a resistivity of $0.5 \Omega \mathrm{cm}$. The piezoresistors are formed using a second epitaxial layer (see figure 1(a)) $500 \mathrm{~nm}$ thick, boron doped with a concentration of $1.1 \times 10^{18}$ atoms $\mathrm{cm}^{-3}$ to achieve a resistivity of $3.75 \times$ $10^{-2} \Omega \mathrm{cm}$. By using the epitaxial growth a uniformly doped layer with a very accurate thickness can be obtained, resulting in uniform and reproducible piezoresistors. The piezoresistors

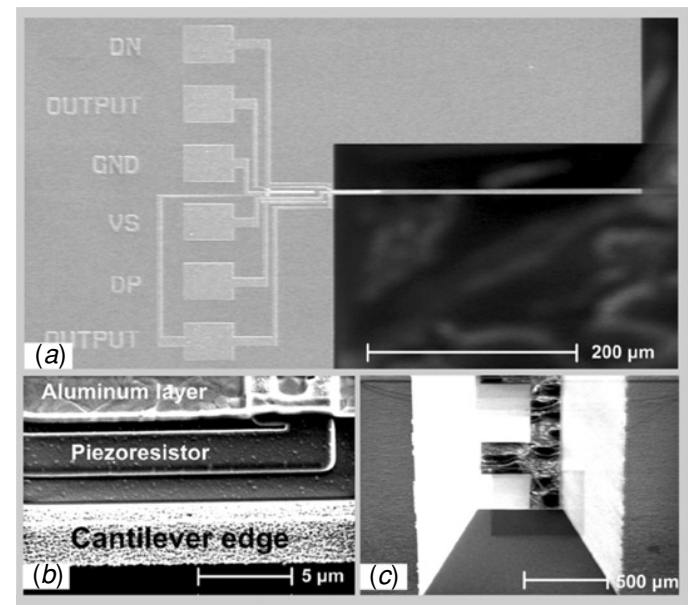

Figure 2. SEM images of a lateral force-sensing piezoresistive cantilever: $(a)$ the complete device; $(b)$ close-up of a piezoresistor; (c) backside view of an etched cavity.

are defined using reactive ion etching (RIE) of silicon as shown in figure $1(b)$. The longitudinal dimension of the piezoresistors is oriented along the [1 110$]$ direction in the $\left(\begin{array}{lll}0 & 0 & 1\end{array}\right)$ plane. They are isolated from the substrate and each other by the n-type epitaxial layer and by implanted regions using boron at $180 \mathrm{keV}$ with a dose of $5 \times 10^{15} \mathrm{~cm}^{-2}$. The contact windows are realized by ion implantation of arsenic $(180 \mathrm{keV}, 5 \times$ $\left.10^{15} \mathrm{~cm}^{-2}\right)$ and boron $\left(15 \mathrm{keV}, 3 \times 10^{15} \mathrm{~cm}^{-2}\right)$ for the substrate and the resistors, respectively.

The next step is the fabrication of the resistors interconnects. First, a $300 \mathrm{~nm}$ thick LPCVD silicon nitride layer is deposited as an electrical insulation layer on the front side. On the backside, it serves at the same time as a masking layer during silicon etching in $\mathrm{KOH}$. After opening the contact windows by a dry etching step of the nitride, a $600 \mathrm{~nm}$ aluminum layer is sputtered and patterned, as shown in figure $1(c)$. The final part of the process (see figure $1(d)$ ) consists of the cantilever definition and release by a combination of wet and dry silicon etching. The bulk silicon is etched from the backside in a $33 \mathrm{wt} \% \mathrm{KOH}$ solution at $85^{\circ} \mathrm{C}$ to create a $10 \mu \mathrm{m}$ thick silicon membrane. The use of a vacuum holder during this etch step avoids any contact between the front side metallization and the $\mathrm{KOH}$ solution. Then the releasing of the cantilever is done by using RIE from the front side to etch the $10 \mu \mathrm{m}$ remaining silicon. A completed $300 \mu \mathrm{m}$ long, $10 \mu \mathrm{m}$ thick and $14 \mu \mathrm{m}$ wide cantilever is shown in figure 2. A close-up of one of the piezoresistors can be seen in figure $2(b)$, while in figure $2(c)$ a backside view of a cavity after etching is shown.

An optical image of the realized piezoresistors is presented in figure 3 . The resistor pair located on the cantilever are the sensing resistors. Their resistance changes when stress is applied on the cantilever. The other two resistors are outside of the cantilever; thus their resistance does not depend on the stress and can be used to compensate for common mode signals in a Wheatstone bridge configuration.

\section{Design}

The resistance change for piezoresistors used in a mechanical sensor can be calculated as the function of the stress at the 


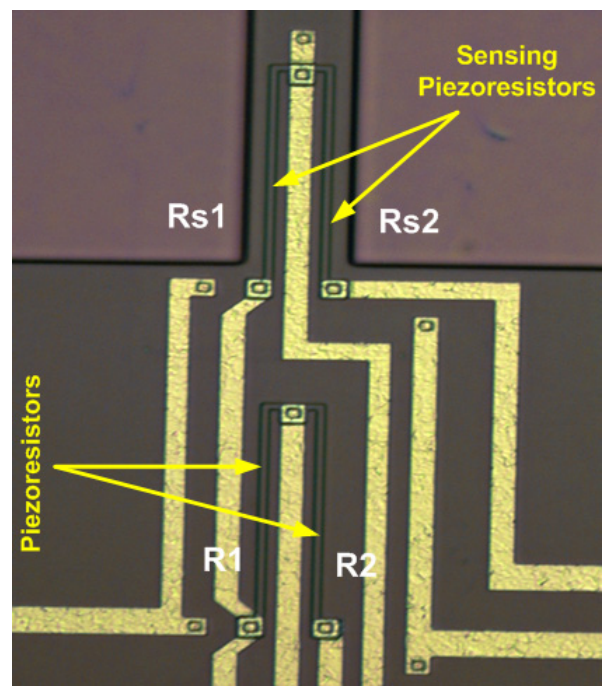

Figure 3. Optical image of the implemented piezoresistors pairs.

surface of the cantilever. Assuming the stress is uniform over the entire piezoresistor, the resistance change due to the mechanical stress is given by [11]

$$
\frac{\Delta R}{R}=\pi_{l} \sigma_{l}+\pi_{t} \sigma_{t}
$$

where $\pi_{l}$ and $\pi_{t}$ are the longitudinal and the transverse piezoresistance coefficients, and $\sigma_{l}$ and $\sigma_{t}$ are the longitudinal and the transverse stresses, respectively. $\sigma_{t}$ can be neglected in a thin cantilever with a small deflection; so equation (1) can be reduced to

$$
\frac{\Delta R}{R}=\pi_{l} \sigma_{l}
$$

The piezoresistive coefficients depend strongly on the doping type, and on the orientation of the stress and the resistor with respect to the crystal axes of silicon. They are a weak function of the doping level for doping concentrations below $10^{19} \mathrm{~cm}^{-3}$. For a resistor and beam along the [1 10$]$ crystal direction, several values of $\pi_{1,110}$ are reported in the literature $[11,12]$.

The lateral and vertical stiffness of the cantilever, $K_{L}, K_{V}$, are given by [11]

$$
\begin{aligned}
K_{L} & =\frac{E W^{3} H}{4 L^{3}} \\
K_{V} & =\frac{E W H^{3}}{4 L^{3}}
\end{aligned}
$$

where $E$ is the modulus of elasticity of the cantilever; $W, H$ and $L$ are the geometrical parameters as depicted in figure 4 . The lateral and vertical stiffness of a $300 \mu \mathrm{m}$ long, $10 \mu \mathrm{m}$ thick, and $14 \mu \mathrm{m}$ wide cantilever are 43 and $22 \mathrm{~N} \mathrm{~m}^{-1}$, respectively.

When a lateral force is applied to the free end of the cantilever, the deflection of the cantilever occurs only in the lateral direction, meaning that the vertical deflection and the transverse sheer stresses are zero. The relationship between the relative change of the piezoresistance and the lateral applied force is given by

$$
\frac{\Delta R}{R}=\frac{12 \pi_{l} F z}{W^{3} H}(L-l / 2)
$$

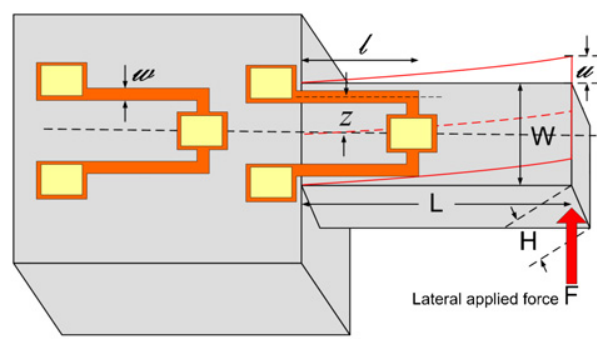

Figure 4. Geometrical parameters of the cantilever and piezoresistor.

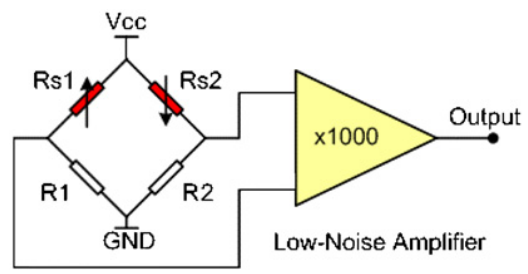

Figure 5. Schematic circuit of the lateral force-sensing piezoresistive cantilever.

where $z$ is the distance from the cantilever neutral axis to the piezoresistor (see figure 4 ) and always smaller than half the cantilever width, $l$ is the length of the piezoresistor, $F$ is the applied force to the tip of the cantilever. Equation (5) indicates that the relative change of resistance of the piezoresistors is directly proportional to the applied force, approximately to the length of the cantilever, and the distance from the neutral axis to the piezoresistor, and inversely proportional to the thickness and cubic width of the cantilever.

Figure 5 shows the resistor connections of the lateral force-sensing piezoresistive cantilever to form a Wheatstone bridge. The bridge has four contacts, two of which provide the dc or ac supply voltage; the others are the output. There are two piezoresistors, Rs1 and Rs2, located on two sides of the cantilever, as illustrated in figure 3 . The resistance of those resistors changes in opposite directions if a lateral force is applied. The other piezoresistors $\mathrm{R} 1, \mathrm{R} 2$ are the common mode compensation resistors. The bridge configuration of the resistors compensates for the signals caused by a vertical deflection. It reduces the cross sensitivity and the offset.

When the piezoresistor is changed by an amount $\Delta R$ the output signal is given by

$$
V_{\text {out }} \approx \frac{V_{\mathrm{cc}} \Delta R}{2 R}
$$

where $V_{\mathrm{cc}}$ is the supply voltage and $R$ is the resistance of the piezoresistor. From equations (5) and (6), and assuming the value of $\pi_{1,110}$ is $59 \times 10^{-11}$ [12], the output is expected to change by $0.075 \mu \mathrm{V}$ when the applied force changes $1 \mathrm{nN}$, which yields a sensitivity of $75 \mathrm{~V} \mathrm{~N}^{-1}$.

\section{Measurements and results}

Figure 6 shows the current-voltage characteristics of the fabricated piezoresistors at room temperature. The characteristic is fairly linear over the voltage range of $20 \mathrm{~V}$, meaning that the contacts and resistors behave as expected. 


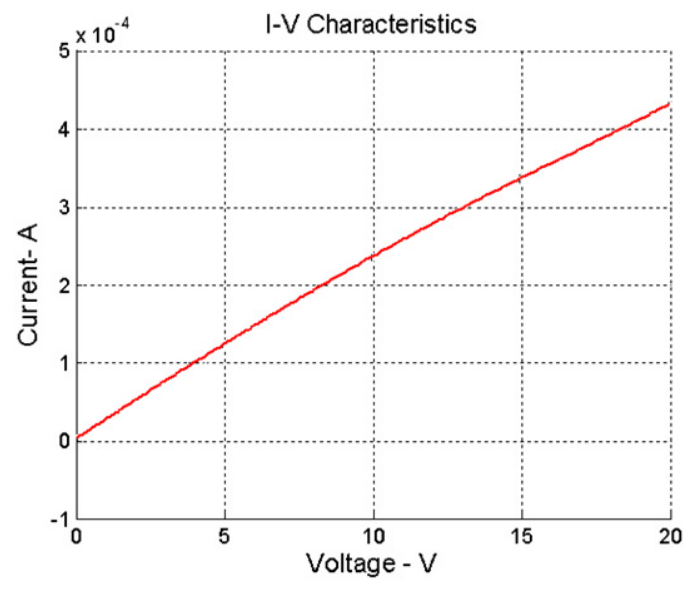

Figure 6. $I-V$ characteristics of the piezoresistors.

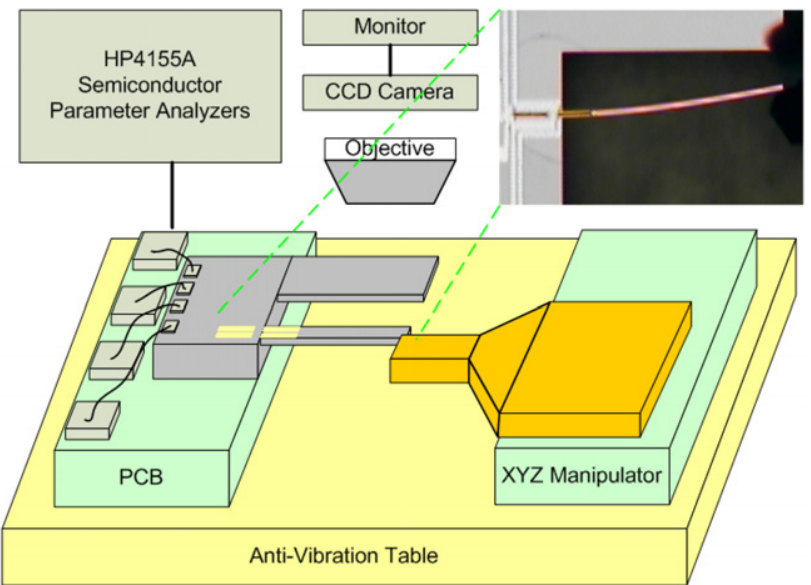

Figure 7. Schematic drawing of the measurement setup. The XYZ manipulator is used to bend the force sensing cantilever. Inset: optical image of a bent beam during measurement.

The zero-stress value of the $73 \mu \mathrm{m}$ long and $1 \mu \mathrm{m}$ wide resistors is $41 \mathrm{k} \Omega$.

In order to apply a force to the tip of the cantilever, the cantilever is bonded to a printed circuit board. A needle, which is fastened to an XYZ manipulator, is used to bend the tip of the cantilever. The cantilever is bent over a small angle, as illustrated in figure 7. By monitoring the deflection we received the response of the piezoresistor.

Figure 8 shows the output voltage due to the deflection of the free end of the cantilever. The bias voltage is $1 \mathrm{~V} \mathrm{dc}$. The graph shows that the relation between the voltage and the applied deflection is fairly linear. The experimental output is $0.16 \mathrm{~V}$ for a deflection of $37 \mu \mathrm{m}$. Therefore the sensitivity is $4.3 \mathrm{kV} \mathrm{m}^{-1}$, meaning that the output voltage changes by $4.3 \mu \mathrm{V}$ when the deflection changes by $1 \mathrm{~nm}$. Figure 8 also shows the effect of the vertical deflection on the cantilever. The sensitivity to the vertical deflection is $24 \mathrm{~dB}$ smaller than the lateral one.

The applied force is given by $F=K u$, where $u$ is the deflection and $K$ is the stiffness of the cantilever. There is a difference between the width and the thickness of the cantilever; hence the lateral stiffness is different from the vertical one. This means that, with the same deflection,

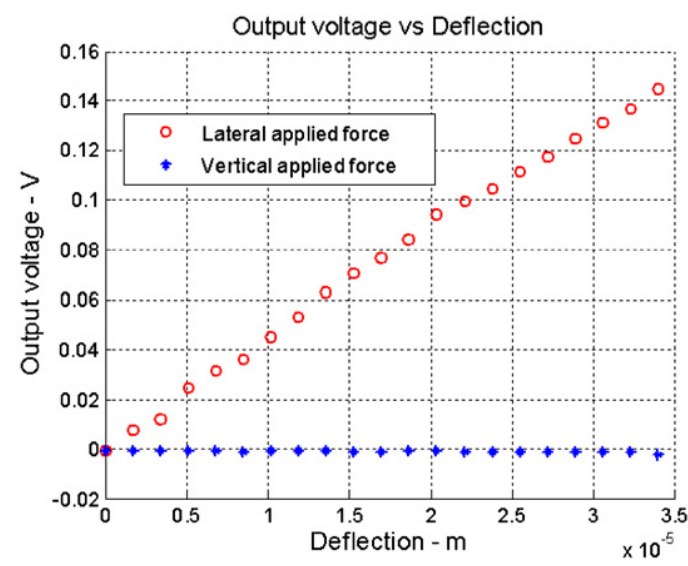

Figure 8. Output voltage due to the deflection of the free end of the cantilever.

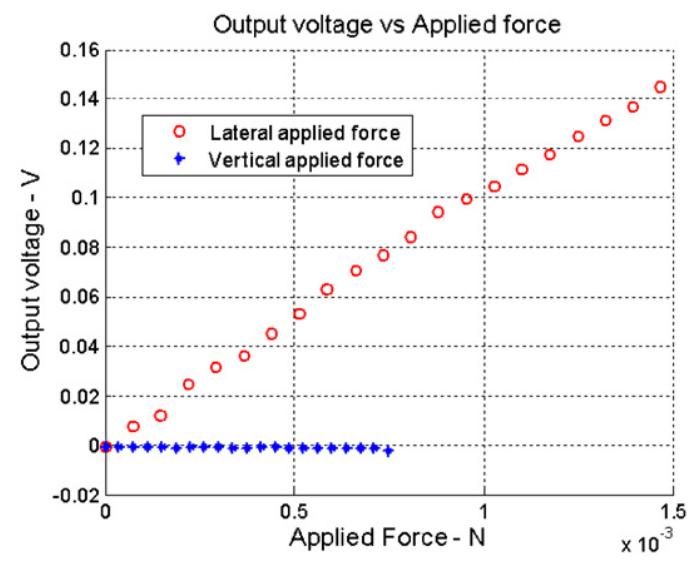

Figure 9. Output voltage due to the applied force.

the applied forces in lateral and vertical cases are different. Figure 9 shows the output signal when a lateral force is applied. The sensitivity derived from this curve is $98 \mathrm{~V}$ $\mathrm{N}^{-1}$, indicating that the epitaxial growth and etching processes behave as expected. This value is somewhat larger than the above mentioned theoretical value of $75 \mathrm{~V} \mathrm{~N}^{-1}$, calculated with the assumption of a $\pi_{1,110}$ value of $59 \times 10^{-11}$.

The two dominant noise sources of the piezoresistive cantilever are Johnson and flicker $(1 / f)$ noise [13]. The Johnson power noise spectral density for a resistance $R$ at a temperature $T$ is given by

$$
S_{J}=4 k_{\mathrm{B}} T R
$$

where $k_{\mathrm{B}}$ is the Boltzmann constant. The flicker power noise spectral density is given by

$$
S_{f}=\frac{\alpha V^{2}}{f N}=\frac{\alpha V^{2}}{c_{i} l w t f}
$$

where $V$ is the bias voltage across a resistor with the total number of carriers $N, f$ is the frequency, and $\alpha$ is a dimensionindependent device parameter which is between $3.2 \times 10^{-6}$ and $5.7 \times 10^{-6}$ in single crystal silicon [13], $c_{i}$ is the charge carrier concentration, $l, w, t$ are the piezoresistor length, width, and thickness, respectively (see figure 4). In single crystal silicon, the flicker noise dominates in the band below $100 \mathrm{~Hz}$. Above this frequency it can be neglected in comparison with the Johnson noise. 
The equivalent noise voltage in a band $\left(f_{\max }-f_{\min }\right)$ is given by

$$
V_{n}=\sqrt{4 k_{\mathrm{B}} T R\left(f_{\max }-f_{\min }\right)+\frac{\alpha V^{2}}{c_{i} l w t} \log \left(\frac{f_{\max }}{f_{\min }}\right)} .
$$

Assuming a band between $1 \mathrm{~Hz}$ and $2 \mathrm{kHz}$, the above equation, and the output signal from figure 9 , the minimum detectable force of the piezoresistive force cantilevers can be estimated as $5 \mathrm{nN}$.

\section{Conclusions}

This paper presents the design, fabrication and characterization of lateral nano-Newton force piezoresistive cantilevers. These cantilevers are based on single crystal silicon wafers instead of the SOI wafers generally employed to achieve high sensitivity force sensors. The proposed structure and its electronic circuit can eliminate the effect of the vertical force, thus realizing a lateral force sensor. The cantilever shows a sensitivity of $98 \mathrm{~V} \mathrm{~N}^{-1}$. Its detection limit is estimated at $5 \mathrm{nN}$. Our force sensor can potentially be used in micro-assembly and biological assays where the force feedback is needed.

\section{Acknowledgments}

The authors would like to acknowledge the whole DIMESICP group for technical support. We also would like to thank W J Venstra, Advanced Mechatronics group, and P J Trimp, Electronic Instrumentation laboratory, for their help with mechanical measurements. This work is partially supported by the Vietnamese Ministry of Education and Training.

\section{References}

[1] Yang C K, Babaei Gavan K, Pandraud G, Katan A J, Van der Drift E and French P J 2005 Proc. 16th
MicroMechanics Europe Workshop (Goteborg, Sweden) pp 316-9

[2] Sarajlic E, de Boer M J, Jansen H V, Arnal N, Puech M, Krijnen G and Elwenspoek M 2004 Advanced plasma processing combined with trench isolation technology for fabrication and fast prototyping of high aspect ratio MEMS in standard silicon wafers J. Micromech. Microeng. $1470-6$

[3] Toriyama T, Tanimoto Y and Sugiyama S 2002 Single crystal silicon nano-wire piezoresistors for mechanical sensors $J$. Microelectromech. Syst. 11 605-7

[4] Onoe H, Gel M, Hoshino K, Matsumoto K and Shimoyama I 2005 Proc. 18th IEEE Conf. on MEMS (Miami, FL, USA) pp 16-9

[5] Baselt D R, Lee G U, Hansen K M, Chrisey L A and Colton R J 1997 A high-sensitivity micromachined biosensor Proc. IEEE $\mathbf{5 8}$ 672-9

[6] Gel M and Shimoyama I 2004 Force sensing submicrometer thick cantilevers with ultra-thin piezoresistors by rapid thermal diffusion J. Micromech. Microeng. 14 423-6

[7] Harley J A and Kenny T W 1999 Proc. 10th Conf. on Solid-State Sensors and Actuators, Transducers (Sendai, Japan) pp 1628-31

[8] Su Y, Evans A G R and Brunnschweiler A 1996 Micromachined silicon cantilever paddles with piezoresistive readout for flow sensing J. Micromech. Microeng. 6 69-72

[9] Chui B W, Kenny T W, Mamin H J, Terris B D and Rugar D 1998 Independent detection of vertical and lateral forces with a sidewall-implanted dual-axis piezoresistive cantilever Appl. Phys. Lett. 92 1388-90

[10] Nanver L K, Goudena E J G and van Zeijl HW 1996 Optimization of fully implanted NPNs for high frequency operation IEEE Trans. Electron Devices 43 1038-40

[11] Senturia Stephen D 2002 Microsystem Design (Boston, MA: Kluwer) pp 469-95

[12] Creemer J F 2002 The effect of mechanical stress on bipolar transistor characteristics $P h D$ Thesis Delft University of Technology (Delft, The Netherlands: Eburon) p 139

[13] Yu X, Thaysen J, Hansen O and Boisen A 2002 Optimization of sensitivity and noise in piezoresistive cantilever $J$. Appl. Phys. 92 6296-301 